Open Access

\title{
Understanding STDMA via computer simulation: feasibility to vehicular safety applications, configurations, and time synchronization errors
}

\author{
Jae-Han Lim
}

\begin{abstract}
Recently, several researchers have proposed to employ self-organized time division multiple access (STDMA) as an alternative Medium Access Control (MAC) protocol of vehicular communications and have studied STDMA to verify its feasibility to vehicular safety applications. However, most of studies have mainly focused on comparing STDMA with enhanced distributed channel access (EDCA) and have several limitations to reveal the feasibility: (1) they have not considered time synchronization error and (2) they have evaluated STDMA with only a single set of configurable parameters. Thus, the feasibility of STDMA to vehicular safety applications still remains unexplored and is an open issue. In this paper, we examine the performances of STDMA to confirm two points: (1) whether or not STDMA is feasible to safety applications even with time synchronization error and (2) whether or not proper configuration really helps to improve the performances. Our simulation results corroborate that current form of STDMA is not feasible to vehicular safety applications and we can improve STDMA via proper parameter configurations.
\end{abstract}

Keywords: Vehicular safety applications, STDMA, Feasibility, Time synchronization error, Configuration

\section{Introduction}

In 2009, the US government reported that more than 33,800 people were killed and more than two million people were injured from vehicle accidents [1]. To reduce the accidents, many academic researchers and car manufacturers have developed active safety systems that relied on vehicular communications. Specifically, vehicles notice the possibility of an accident by exchanging their status information (e.g., position, speed, and acceleration), thereby being able to prepare for the accident before it happens.

Enhanced distributed channel access (EDCA) is a default Medium Access Control (MAC) protocol of dedicated short-range communications (DSRC), which is the de facto standard for vehicular communications [2]. The core mechanism of EDCA is carrier sense multiple access with collision avoidance (CSMA/CA), which

Correspondence: ljhar@etri.re.kr

Electronics and Telecommunications Research Institute, 218 Gajeong-ro, Yuseong-gu, 34129 Daejeon, Korea controls multiple access in a distributed manner. The distributed nature of CSMA/CA makes EDCA robust to frequent topology changes in vehicular networks. However, EDCA is not suitable to vehicular safety applications, which require high delivery ratio and low latency [3]. This is because the random nature of CSMA/CA causes unpredictable delay, which leads to frequent violation of latency requirement. Even more, CSMA/CA is prone to packet collisions, which decrease delivery ratio $[4,5]$.

It is well-known that time division multiple access (TDMA) can address the challenges of EDCA. Specifically, when employing TDMA for vehicular communications, the upper bound of delay can be guaranteed and packet collisions rarely happen even in high vehicle density due to a well-organized time schedule [6,7]. However, for organizing time schedule well, we require a central controller (e.g., road side unit (RSU)), of which implementation and management is costly.

To address the challenges of EDCA and TDMA, several researchers have proposed to adopt Self-organized TDMA (STDMA) as an alternative MAC for vehicular 
communications [9-11]. STDMA has both deterministic and distributed natures. More specifically, each vehicle transmits a packet according to a time slot schedule (deterministic) and chooses its own time slot by itself (distributed). Thus, STDMA seems to be feasible to vehicular safety applications.

Even if there have been previous simulation studies when STDMA was used for vehicular networks [9-14], they have mainly focused on comparing network performances of CSMA/CA with those of STDMA and have concluded that STDMA was more suitable to vehicular networks than CSMA/CA. But, these studies could not confirm the feasibility of STDMA to vehicular safety applications because they have several missing points(1) no consideration of time synchronization error and (2) evaluation with a single set of configurations. We will explain why considering these two points are critical for feasibility study and improvement of STDMA in the following paragraph.

First, the previous studies have been based on unrealistic assumption that practical errors (e.g., time synchronization error) did not occur in vehicular communications. However, time synchronization error often occurs when losing global positioning system (GPS) signal; this error can degrade network performances of STDMA. Thus, we cannot confirm the feasibility of STDMA just using the simulation results of the previous studies. Second, previous studies have considered only a single set of configurations, even though STDMA is often associated with MAC and PHY configurable parameters. Hence, even if previous studies have shown that STDMA was not feasible to vehicular safety applications, there is room for improvement by configuring STDMA parameters carefully. Thus, we must verify that proper configurations really help to improve STDMA performance. Moreover, we emphasize that understanding the impact of each configurable parameter can provide rich set of hints for configuring parameters to improve STDMA. More specifically, we must show clearly which performance measure (e.g., packet delivery ratio (PDR), delay) is associated with each parameter.

At this point, we can raise two important questions: “(1) Can STDMA support vehicular safety applications even with the practical error and (2) proper configurations contribute to improvement of STDMA?" To answer these questions, in this paper, we conduct in-depth simulation studies on STDMA as follows. First, we study impacts of time synchronization errors on STDMA performances. Second, we investigate STDMA performances by varying several system parameters.

Simulation results demonstrate that STDMA with typical parameter settings $[8,9]$ is limited to be used for vehicular safety applications and can be enhanced via proper configurations. Then, we investigate what are mainly attributed to such limitations and suggest guidelines for improving STDMA to fit in with vehicular safety applications. In Table 1, we summarize key findings on (1) feasibility of STDMA to vehicular safety applications (Feasibility) and (2) hints for suitable configurations (Configuration). We emphasize that our study will help to understand the characteristics of STDMA and our findings will pave the way for improving the current form of STDMA.

The contributions of this paper are three-folds.

- We analyze STDMA performances with practical errors that can occur in real worlds (e.g., time synchronization error) and evaluate the feasibility of STDMA to vehicular safety applications in such situations. We confirm that the current form of STDMA is not feasible to vehicular safety applications.

- We study the impact of configurable parameters, confirm that proper setting of each configurable parameter really helps to improve STDMA performance, and provide a set of hints for configurations based on observations.

- We suggest guidelines for STDMA improvement to be suitable to vehicular safety applications.

The rest of this paper is organized as follows. In Section 2, we introduce previous works on STDMA evaluation. In Section 3, we briefly explain STDMA. In Section 4, we evaluate STDMA thoroughly and verify the feasibility of STDMA to vehicular safety applications. In Section 5, we provide several suggestions for improving STDMA based

Table 1 Summary of key findings in our study

\begin{tabular}{|c|c|c|}
\hline Category & Findings & Sections \\
\hline Configuration & $\begin{array}{l}\text { SI rarely affects PDR and } \\
\text { delay of STDMA }\end{array}$ & Section 4.3 \\
\hline Configuration & $\begin{array}{l}\text { Physical transmission rate } \\
\text { significantly affects PDR. } \\
\text { A rate maximizing PDR is } \\
\text { likely to increase with } \\
\text { vehicle density }\end{array}$ & Section 4.4 \\
\hline Configuration & $\begin{array}{l}\text { Proper configuration of } \\
\text { CS threshold helps to } \\
\text { improve PDR }\end{array}$ & Section 4.5 \\
\hline Configuration & $\begin{array}{l}\text { Delay is independent of SI, } \\
\text { CS threshold, and physical } \\
\text { transmission rate }\end{array}$ & Sections $4.3,4.5,4.4$ \\
\hline Configuration & $\begin{array}{l}\text { Delay of STDMA decreases } \\
\text { linearly with NI. }\end{array}$ & Section 4.6 \\
\hline Feasibility & $\begin{array}{l}\text { PDR is not high enough } \\
\text { to support critical safety } \\
\text { applications, particularly in } \\
\text { high vehicle density }\end{array}$ & Section 4.7 \\
\hline Feasibility & $\begin{array}{l}\text { Time synchronization error } \\
\text { degrades PDR }\end{array}$ & Section 4.7 \\
\hline
\end{tabular}


on simulation findings. In Section 6, we conclude our work.

\section{Related work}

\subsection{Mechanisms for improving vehicular networks}

Many researchers have made effort to improve performances of vehicular networks by proposing several mechanisms. In [33] and [39], authors have proposed routing algorithms to enhance performances of vehicular networks. The authors in [28, 30, 34] have provided guidelines that helps to improve routing mechanisms in vehicular networks. The authors in [29] focused on delay-constraint topology control problem and proposed interference-based topology control mechanism. In [32], a resource allocation mechanism was proposed in selforganizing LTE, which might be useful when LTE is adopted for inter-vehicle communications.

Many researchers have attempted to enhance security of vehicular networks [31, 37, 38, 41, 43, 44, 46]. In [31], the authors focused on security problems in Internet of Things (IoT), in which vehicles are one of main elements. The authors in [38] introduced a threshold credit-based incentive mechanism to make devices robust to compromise attacks in cloud-assisted vehicular delay tolerant network (DTNs). In [37, 41, 43, 44, 46], the authors have proposed key security algorithms that can be applied to vehicular networks. Moreover, some researchers have broaden the spectrum of possible applications of vehicular communications: multimedia services [35] and cloud computing $[36,42,45]$.

\subsection{Simulation studies on EDCA and STDMA for vehicular networks}

Many researchers have confirmed via computer simulation that EDCA could not satisfy stringent delay and PDR requirements of safety services in high vehicle density. Simulation studies in $[24,25]$ have demonstrated that network congestion was mainly attributed to low PDR of EDCA. In [4], the authors showed via simulation that the current form of EDCA was not suitable to vehicular safety messaging and proposed two mechanisms for improving EDCA: access category (AC) isolation and virtual division. In [20], the authors conducted experimental studies on DSRC in a real driving situations from a perspective of network engineers. In [22, 23], the authors argued that EDCA had limitations to support safety message dissemination if only a DSRC band was used and have proved the limitations via computer simulations. Then, they have proposed to exploit both DSRC band and TVWS band.

There have been previous simulation studies when STDMA is used for vehicular networks [9-14]. In [9], the authors focused on throughput of STDMA via simulation studies. In [10], the authors analyzed the real-time properties of CSMA/CA and STDMA via simulation studies in highway scenarios and revealed that STDMA was better than CSMA/CA. The authors in [11] pointed out that CSMA/CA was not suitable to vehicular safety applications and showed that STDMA outperformed CSMA/CA. In [12], the authors focused on the hidden terminal problem when using CSMA and STDMA for vehicular communications. The authors in [13] conducted simulation studies on CSMA/CA and STDMA in terms of MAC delay with two message settings: 300 bytes $/ 10 \mathrm{~Hz}$ and 800 bytes $/ 2 \mathrm{~Hz}$. In [14], the authors made efforts to reveal when slot allocation collisions happen in STDMA. However, previous works have mainly focused on comparing performances of CSMA/CA with those of STDMA when applying them to vehicular beaconing and they have not considered important factors to truly understand the characteristic of STDMA: (1) impact of time synchronization error and (2) impact of several configurable parameters. Thus, we need to develop a quantitative understanding of STDMA in realistic situations by considering all missing points of the previous studies.

\section{Self-organized time division multiple access (STDMA)}

Self-organized time division multiple access (STDMA) is a deterministic MAC protocol, whereby vehicles can access wireless medium at their designated time slots. In STDMA, vehicles determine their own transmission schedules by themselves (i.e., select their own time slots) based on channel measurements. As shown in Fig. 1, time is divided into frames and the frame is further divided into multiple time slots.

STDMA is composed of four phases: (1) initialization phase, (2) network entry phase, (3) first frame phase, and (4) continuous operation phase. In an initialization phase, vehicles listen to wireless channel and store channel activities during one time frame (e.g., the occupation of each slot and a position of the slot owner). In an entry phase, vehicles select their first time slots within a frame, which is denoted by nominal transmission slot (NTS). The details of selecting a time slot will be explained in the following paragraph. In the first frame phase, the vehicle selects time slots for the rest of the frame using the mechanism similar to that used in the network entry phase. The last phase of STDMA is a continuous operation phase, where vehicles exploit NTSs that are chosen in the first frame phase. However, to adapt to network topology change, vehicles select new NTSs after $n$ frames.

The mechanism for selecting time slot (i.e., NTS) in the network entry phase consists of four steps. First, a vehicle calculates a nominal increment (NI) by dividing an average report interval with the unit slot length. Here, the report interval refers to an period that each message must be transmitted and the interval is equal to beacon interval (BI) in vehicular safety applications. Second, a vehicle 


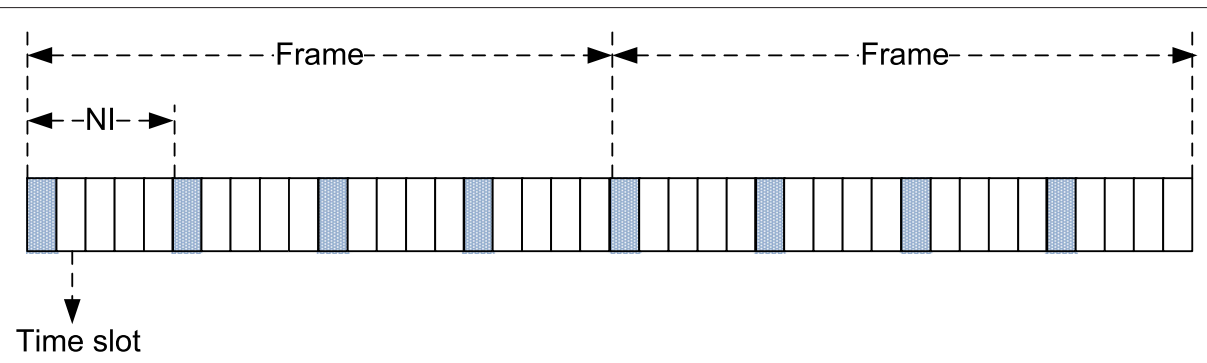

Fig. 1 Time frame structure of STDMA

randomly chooses nominal start slot (NSS) from current slot up to NI. Third, a vehicle sets selection interval (SI) around NSS and randomly picks its NTS within SI. Finally, a vehicle checks an availability of NTS. More specifically, if the chosen NTS is already occupied by someone else based on the previous measurements, a vehicle selects the closest unoccupied slot within SI. However, if all slots within SI are occupied, the vehicle selects the slot of which owner is furthest away from itself. This is because an amount of interference tends to be smaller as the distance from the interferer is getting longer.

\section{In-depth analysis of STDMA using simulator}

In this section, we perform an in-depth simulation study of STDMA using NS-2 simulation [15]. In this study, we isolate the impact of time synchronization errors and the impact of configurable parameters. First, we investigate STDMA according to configurable parameters (i.e., SI, NI, CS threshold, and a physical transmission rate) to get insight on how to configure each parameter for performance enhancement. To isolate the effect of each parameter, we change one parameter while fixing others to default settings and assume that there are no time synchronization errors. Next, we focus on the impact of time synchronization errors, which can happen in practical operations.

\subsection{Simulation setup}

Figure 2 illustrates a topology used in our simulation study. In the study, we consider a two-way two mile road segment with four lanes as we can find in many highways. To model vehicle mobility, we adopt a car-following model proposed by Gipps [26]. We summarize default simulation settings in Table 2. For PHY layer protocol, we follow IEEE 802.11p [4]. In MAC layer setting, we use STDMA system parameters that are calculated from message size (345 bytes), message generation interval $(100 \mathrm{~ms})^{1}$ and physical transmission rate ( $3 \mathrm{Mbps})$. More specifically, the size of unit time slot is derived by dividing a message size by physical transmission rate $[10]^{2}$. Then, we can obtain NI by dividing an average report interval (e.g., beacon interval) by the size of a unit time slot. Finally, we calculate $\mathrm{SI}$ based on $\mathrm{NI}^{3}$. The noise power in our study is $-104 \mathrm{dBm}$, which is derived from Bolzman constant, temperature, and bandwdith ${ }^{4}$. We use Nakagami fading model, which is well-known to be suitable to describe radio propagation in vehicular communications [40].

\subsection{Performance measures}

We exploit two important performance measures for vehicular safety applications: packet delivery ratio (PDR) and communication delay [3]. The two performance measures are defined as follows.

- Packet delivery ratio (PDR): a ratio of the number of vehicles that receive a packet $\left(n_{\text {receive }}\right)$ to the number of vehicles within a transmission range of a sender ( $\left.n_{\text {target }}\right)$.

- Communication delay: an interval between a packet generation time and a packet reception time.

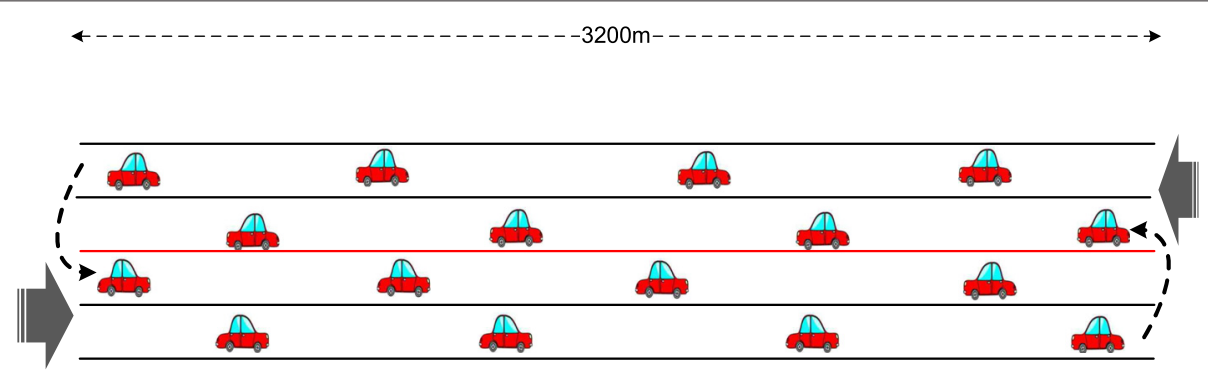

Fig. 2 Topology used in simulation study 
Table 2 Default system parameters

\begin{tabular}{ll}
\hline Parameter & Value \\
\hline Average transmission range & $500 \mathrm{~m}$ \\
Physical transmission rate & $3 \mathrm{Mbps}$ \\
Channel model & Nakagami fading model \\
Message generation & 345 bytes $/ 100 \mathrm{~ms}$ \\
Number of vehicles & Variable \\
Noise power (dBm) & $-104 \mathrm{dBm}$ \\
Parameters for STDMA & $(\mathrm{Nl}, \mathrm{Sl}$, slot size $)=(90,18,1.1 \mathrm{~ms})$ \\
\hline
\end{tabular}

We should note that we do not consider packet losses caused by buffer overflow in calculating PDR. In reality, the overflow rarely happens when we adopt STDMA for broadcasting a vehicular safety message. Specifically, vehicles can transmit their packets at least once every NI, which is comparable to packet generation interval. This implies that the number of packets in the queue is no more than one, which is much smaller than the typical queue size. Thus, buffer overflow does not happen when we employ STDMA.

\subsection{Impact of service interval}

In Fig. 3, we observe that PDR of STDMA does not vary with $\mathrm{SI}^{5}$, which is somewhat counter-intuitive. In STDMA, packet collisions happen when more than two vehicles in a close vicinity select the same time slot; the probability of selecting the same slot rarely changes with SI due to the following reason. For selecting the same slot, two vehicles must satisfy the following two conditions:
(1) SIs of two vehicles must overlap and (2) two vehicles must select the same slot within the overlapping part. The first event becomes more probable as SI rises, whereas the probability of the second event decreases with the rise in SI. As a result, we conclude that the probability of selecting the same slot by two vehicles is rarely affected by SI. ${ }^{6}$

The delay of STDMA does not change with SI, as depicted in Fig. 4. This is because the dominant factor of the delay is queuing delay, which is determined by the message generation time and vehicle's designated time slot. It is obvious that the message generation time is independent of SI. Moreover, the vehicle's designated time slot is rarely affected by the size of SI. Hence, the delay is not affected by the size of SI.

\subsection{Impact of physical transmission rate}

Even if the physical layer of vehicular communications (i.e., IEEE 802.11p) supports multiple physical transmission rates, only the single rate (e.g., $3 \mathrm{Mbps}$ ) has been employed in previous simulation studies $[4,5,9-14]$. However, we expect that PDRs of STDMA could be affected by the transmission rate and proper selection could improve the PDRs. Hence, in this subsection, we will confirm our hypothesis by evaluating the PDRs of STDMA with various physical transmission rates. To understand the impact of the transmission rate clearly, we will further describe the physics of how the rate affects PDRs of STDMA in the following paragraph.

It is well-known that a failure to receive packets is mainly attributed to two factors: (1) packet collisions and

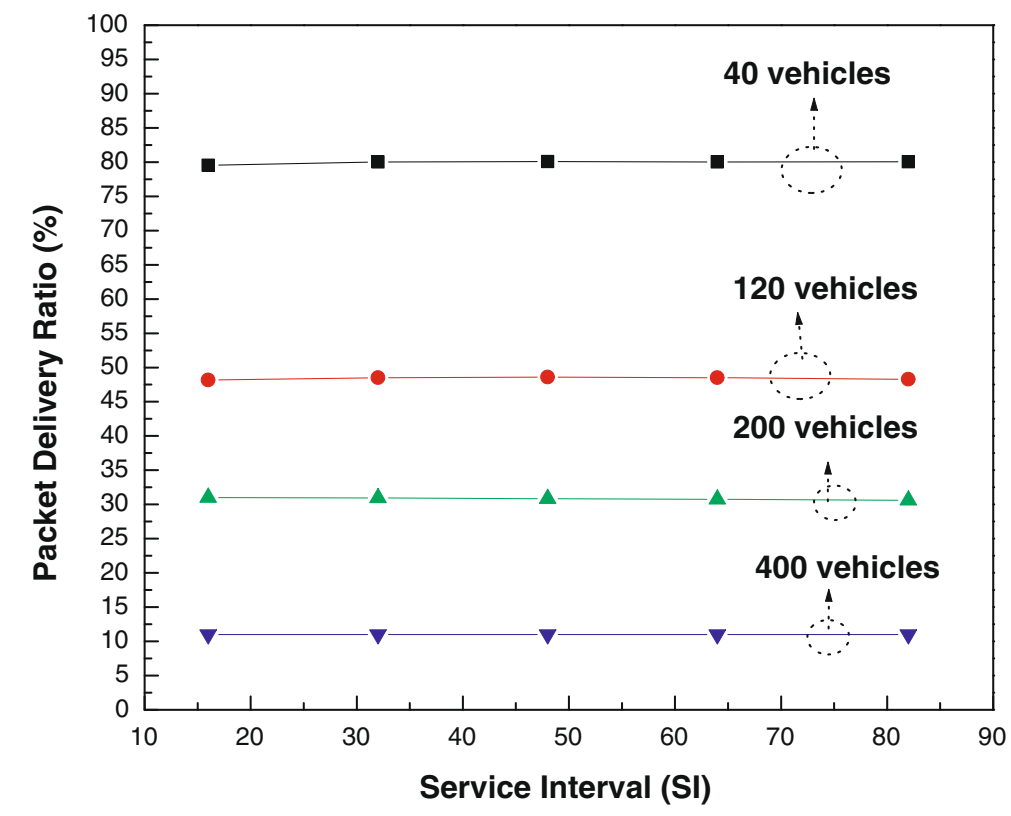

Fig. 3 PDR of STDMA according to service interval (SI) when the number of vehicles is 40, 120, 200, and 400 


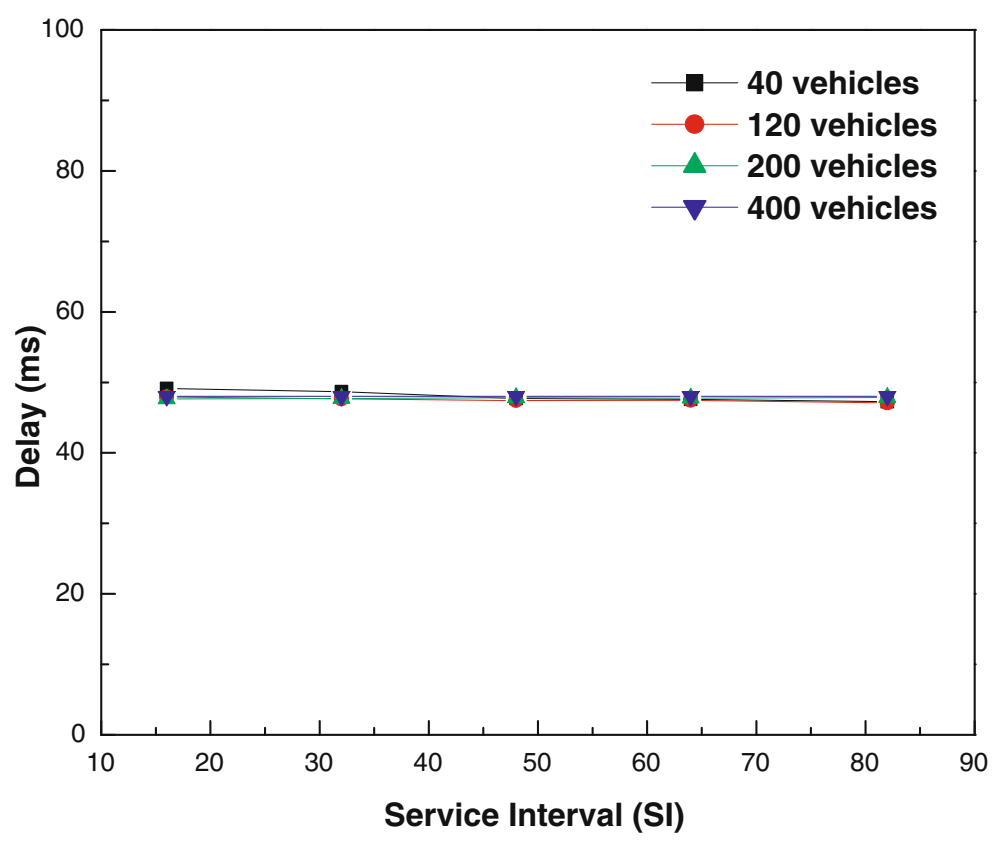

Fig. 4 Delay of STDMA according to service interval (SI) when the number of vehicles is 40, 120, 200, and 400

(2) decoding failure due to channel error, which can be quantified with the probability of collision and the probability of decoding error, respectively. First, the probability of collision decreases with the rate and vice versa. This is because we can include more time slots within NI as the rate increases. ${ }^{7}$ Recall that packet collision happens when more than two vehicles in a close vicinity select the same time slot; thus, the increase in the number of slots within NI reduces the collision probability. Second, it is obvious that the probability of decoding error rises with the transmission rate.

Figure 5 demonstrates that the lowest transmission rate is not always optimal in terms of PDR. Specifically, when the number of vehicles is small, a low transmission rate tends to become optimal (e.g., $6 \mathrm{Mbps}$ when there are 40 and 80 vehicles). This is because a decoding failure is a dominant factor of reception failures rather than a packet collision in low vehicle density. Thus, the adoption of lower transmission rate leads to higher PDR. On the contrary, when the number of vehicles is large, a high transmission rate is normally an optimal rate in terms of PDR (e.g., $18 \mathrm{Mbps}$ when there are 400 vehicles). ${ }^{8}$ This is because a packet collision is a dominant factor rather than a decoding error in high vehicle density. Notably, even if the optimal rate ranges from 6 to $18 \mathrm{Mbps}$ in Fig. 5, transmission rates outside this range could become an optimal rate. For example, the optimal rate could fall down to $3 \mathrm{Mbps}$ when channel conditions become worse (e.g., when large amount of multi-path fading and serious shadowing by obstacles occurs) in low vehicle density.
Moreover, the optimal rate could go up to $27 \mathrm{Mbps}$ when channel conditions become better in high vehicle density.

Similar to Section 4.3, the delay is independent of the transmission rate, as depicted in Fig. 6. It is obvious that a queuing delay accounts for a large portion of the delay; the queuing delay is determined by (1) the message generation time and (2) time assigned for each vehicle, both of which are independent of the transmission rate. Hence, the delay does not depend on the transmission rate.

It is noted that delays of STDMA are independent of the number of vehicles while PDR decrease with the number. This is because delay is mainly attributes to queuing delay, which is determined by (1) message generation time and (2) time assigned for each vehicle, both of which are independent of the number of vehicles. In contrast, PDR is significantly affected by the number of vehicles because the probability of packet collision rises with the number of vehicles. More specifically, packet collision happens when more than two vehicles choose the same time slot; the probability that more than two vehicles choose the same slot increases with the number of vehicles.

\subsection{Impact of carrier sensing threshold}

In Fig. 7, we observe that PDR of STDMA increases with a carrier sensing (CS) threshold until the threshold reaches crossover points (e.g., $-84 \mathrm{dBm}$ in 40 vehicles) and decreases as the threshold passes these points. Figure 8 demonstrates that delays of STDMA are independent of a CS threshold. In the following paragraph, we will further 


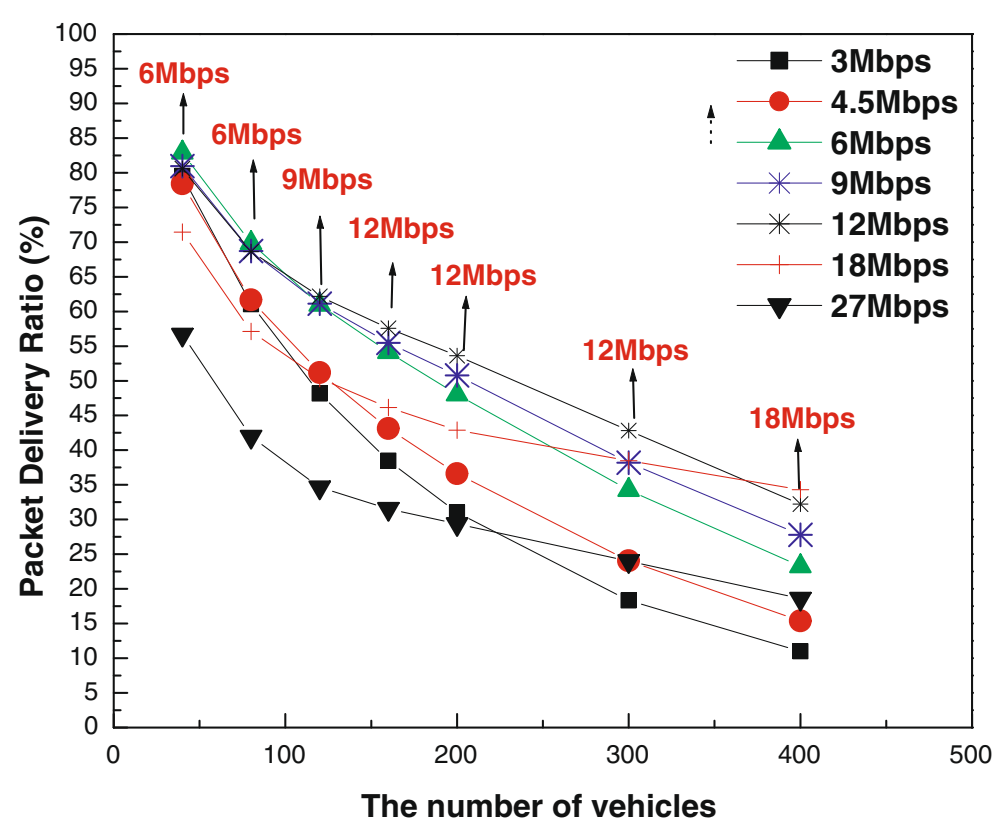

Fig. 5 PDR of STDMA according to the number of vehicles when various physical transmission rates are used. The text pointed by each arrow is a physical transmission rate that maximizes PDR in each "the number of vehicles" setting

explain which factors are mainly attributed to the PDR pattern in more detail.

Vehicles become insensitive in signal detection as CS threshold grows and such insensitivity helps to improve PDR if CS threshold is less than a crossover point. Recall that a vehicle records whether or not each slot is idle in its monitoring table and selects its transmission slot among all the idle slots recorded in the table. Obviously, the insensitivity increases with CS threshold; thus, the number of idle slots recorded in the table increases with a CS threshold. This implies that a probability that more than two vehicles selects the same transmission slot

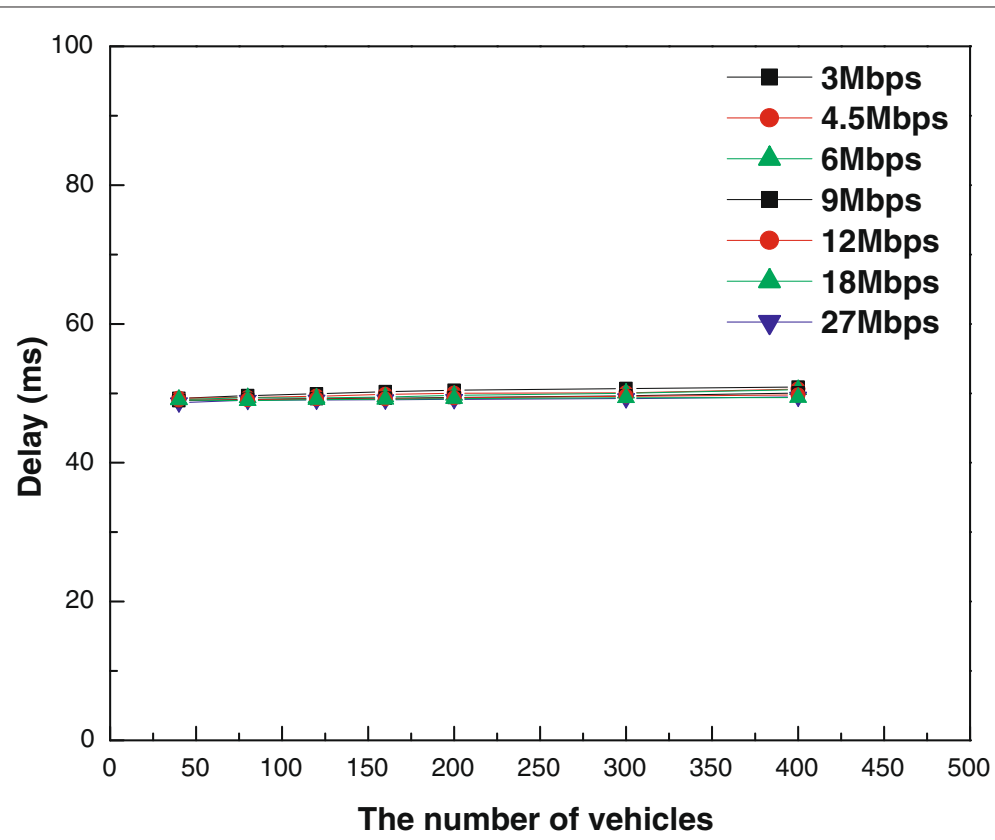

Fig. 6 Delay of STDMA according to the number of vehicles when various physical transmission rates are used 


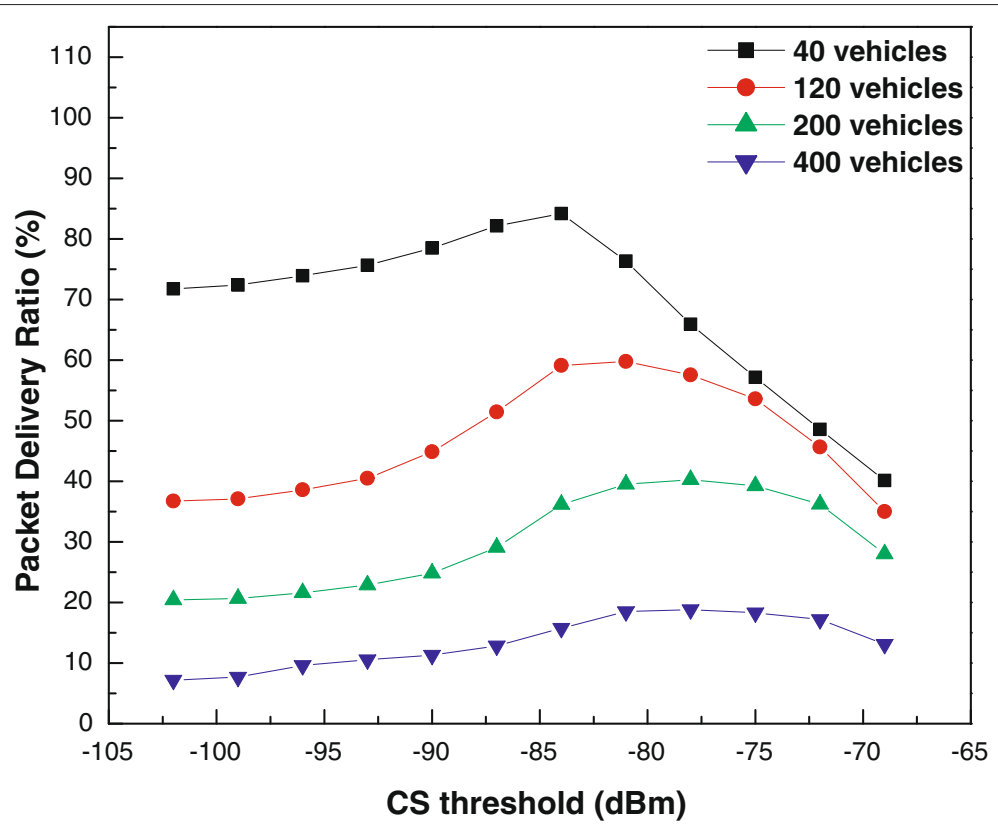

Fig. 7 PDR of STDMA according to the CS threshold when the number of vehicles is 40, 120, 200, and 400

decreases as a CS threshold rises. In contrast, the insensitivity in signal detection contributes to PDR degradation if CS threshold passes the crossover point. In most wireless communication systems, receivers first detect a signal of a packet, then decode the packet [21]. In the regime whereby CS threshold is too high, receivers fail to detect a large fraction of packets. In other words, the detection failure is a dominant factor that influences PDR in this regime; thus, PDR degrades with CS threshold.

We learn two important lessons from these observations as follows. First, we necessarily find an optimal CS threshold to improve PDR and the optimal threshold is different according to the number of vehicles (e.g., $-84 \mathrm{dBm}$ in 40 vehicles and $-81 \mathrm{dBm}$ in 120 vehicles). Thus, we need to

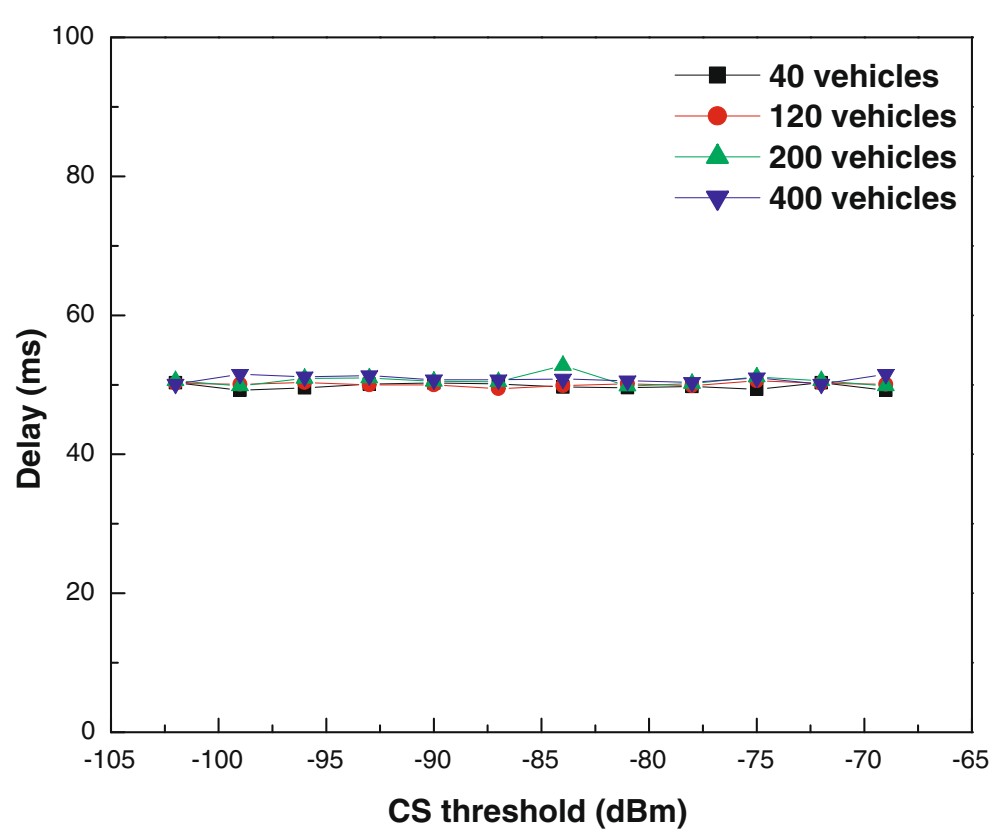

Fig. 8 Delays of STDMA according to the CS threshold when the number of vehicles is 40, 120, 200, and 400 
devise a mechanism that adapts CS threshold to a vehicle density. Second, we cannot reduce a delay by tuning a CS threshold.

\subsection{Impact of nominal increment}

In previous subsections, we observe that delays are unsatisfactory for vehicular safety services even with various configurations (e.g., $50 \mathrm{~ms}$ in Figs. 4, 6, and 8. Fifty milliseconds is independent of SI, a physical transmission rate, CS threshold, and the number of vehicles.). We must note that the delay observed in the previous subsections are almost half of nominal increment (NI) (e.g., we configure NI to be comparable to $100 \mathrm{~ms}$ ). Thus, we expect that delays of STDMA can be reduced via proper configuration of NI, and we will validate our hypothesis in this subsection. Before showing simulation results, we will explain (1) how NI is configured to be less than traffic generation interval and (2) how vehicles select their time slots with this configuration in the following two paragraphs.

STDMA protocol recommends to set NI to be comparable to average packet generation interval $[8,9]$. For example, we often configure NI to be comparable to beacon interval (e.g., $100 \mathrm{~ms}$ ) in vehicular safety services. To reduce the physical duration of NI, we decrease the number of time slots comprising NI rather than reduce the unit slot size. This is because the size is decided by a transmission rate, which must be isolated from NI to clearly understand the effect of NI. For example, in this subsection, we configure NI to be comparable to $99 \mathrm{~m}$ and $33 \mathrm{~ms}$, by setting the unit slot size to be $1.1 \mathrm{~ms}$ and the number of slot to be 90 and 30 , respectively.

A principle to assign a time slot is that each vehicle can transmit its packet once every generation interval while reducing delay as much as possible. For this purpose, we include one more step (i.e., check a message queue before transmission) and do not follow the suggestion of the current protocol [8, 9] about configuration of NI. Specifically, vehicles decide NI independent of generation interval and select their own slots every NI. At every time slot, they check whether to have packets to send in their TX queues. If having packets to send, they transmit their own packets; otherwise, despite having right for channel access (i.e., its turn to transmit), they just monitor the channel rather than transmit their packets.

In Fig. 9, we notice that the delay of STDMA is significantly reduced by changing NI from 90 to 30 . To be specific, the average delay is around $16 \mathrm{~ms}$ when NI is 30 (i.e., physical duration is $33 \mathrm{~ms}$ ), whereas the delay is around $50 \mathrm{~ms}$ when NI is 90 . Here, we must note two points. First, the delay is upper-bounded by NI. This is because every vehicle can have at least one chance to transmit its packet, i.e., at least one slot is assigned, within NI. Second, the average delay is half of NI, which is strikingly similar to results in previous subsections.

\subsection{Impact of time synchronization errors}

In STDMA, time synchronization is important since synchronization error causes slot misalignment, which can induce additional packet collisions. To be specific, even if two vehicles in the vicinity select different time slots, the slot misalignment might cause overlap between their transmissions. Obviously, this overlap causes packet collision, which degrades network performance. Thus, we need to study STDMA performance when time synchronization error exists.

In practical situations, time synchronization error happens when vehicles fail to receive global positioning system (GPS) signal due to large obstacles (e.g., bridges). More specifically, when failing to receive GPS signal, vehicles cannot update its local time using clock info in GPS signal; thus, they fail to align local time clocks to global GPS clock. In this case, vehicles suffer from time synchronization error since local clock generators among vehicles have slight difference. In our simulation study, we assume that vehicles fail to receive GPS signal when located under the bridge and suffer from time synchronization errors with a $100 \mathrm{ppm}$ clock skew rate [19]. Regarding bridge placement, we consider a wide bridge with 10 lanes, which can be found in I-405 freeway area in Los Angeles (http:// maps.google.com).

Figure 10 shows that the time synchronization error induces additional packet collision in STDMA. Specifically, PDR is getting smaller as the number of bridges increases. This is because reception errors of GPS signal becomes more frequent as the number increases. Thus, slot misalignment induces additional packet collisions, thereby reducing PDR.

\section{Discussion}

\subsection{Limitations of current STDMA}

In previous section, we observe that our simulation results on PDR are so disappointing that a current form of STDMA is not feasible to vehicular safety applications [3]. Here, the current form of STDMA is referred to as STDMA following configurations suggested in [8] (e.g., $\mathrm{NI}$ is equal to traffic generation interval, $\mathrm{SI}$ is one fifth of $\mathrm{NI})$. For example, as depicted in Fig. 10, PDR is around $30 \%$ when 400 vehicles are deployed in a $2 \mathrm{mi}$ road; PDR becomes smaller when those vehicles suffer from time synchronization error. In addition, we notice that delay of STDMA is less satisfactory than is required to support critical safety applications with typical configurations of NI. For example, pre-crash sensing requires communication delay less than $20 \mathrm{~ms}$ according to [3]. However, Figs. 4, 6, and 8 demonstrate that average delays are $50 \mathrm{~ms}$ when we configure NI to be equal to typical beacon interval, i.e., $100 \mathrm{~ms}$.

From these observations, we can conclude that the current form of STDMA is not feasible to vehicular safety 


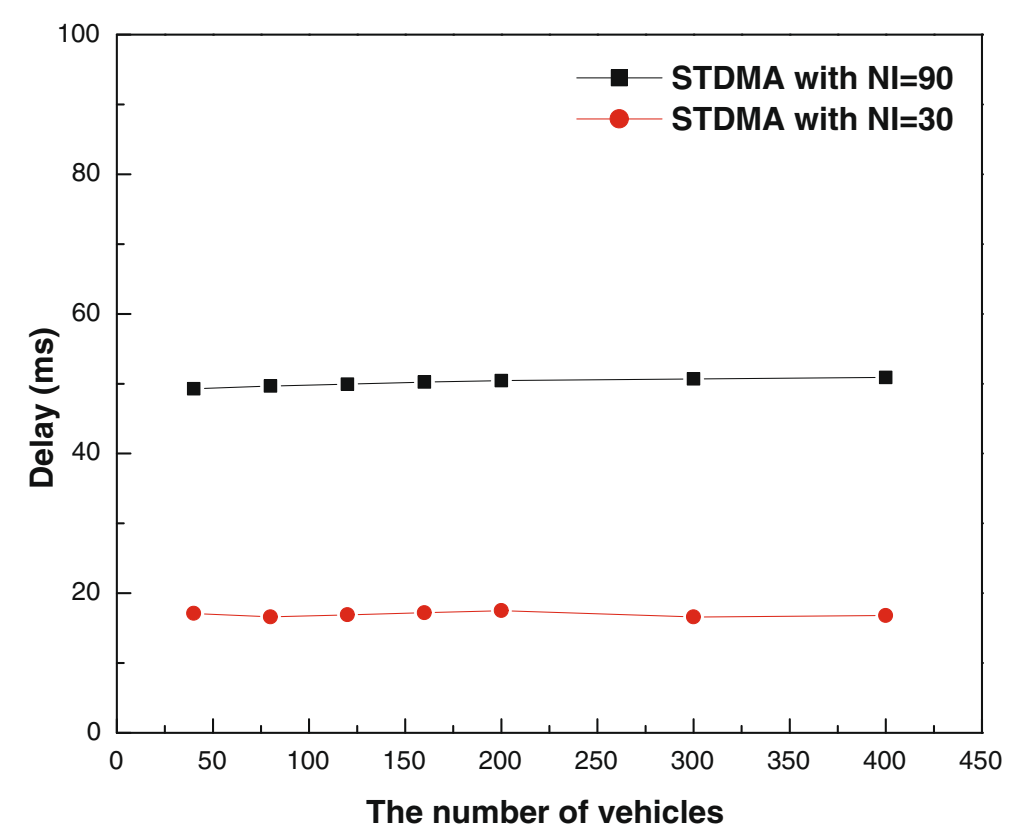

Fig. 9 Delay of STDMA according to number of vehicles when nominal increment (NI) is 30 and 90

applications. Thus, it is crucial to improve STDMA particularly when there are a large number of vehicles. To understand the limitations of current STDMA clearly, we will describe what are attributed to low PDR and long delay in the following two paragraphs.

The reasons of low PDR are threefolds. First, STDMA lacks an organized schedule in time slot assignment. As we mentioned in Section 3, a vehicle selects its own time slot in the network entry phase and several steps comprising the phase include random selection. Specifically, NSS (or NS) is selected randomly within NI (second step), and time slot is selected randomly among idle slots within SI (third step). It is obvious that vehicles often select the same slot due to these random selections, and we expect

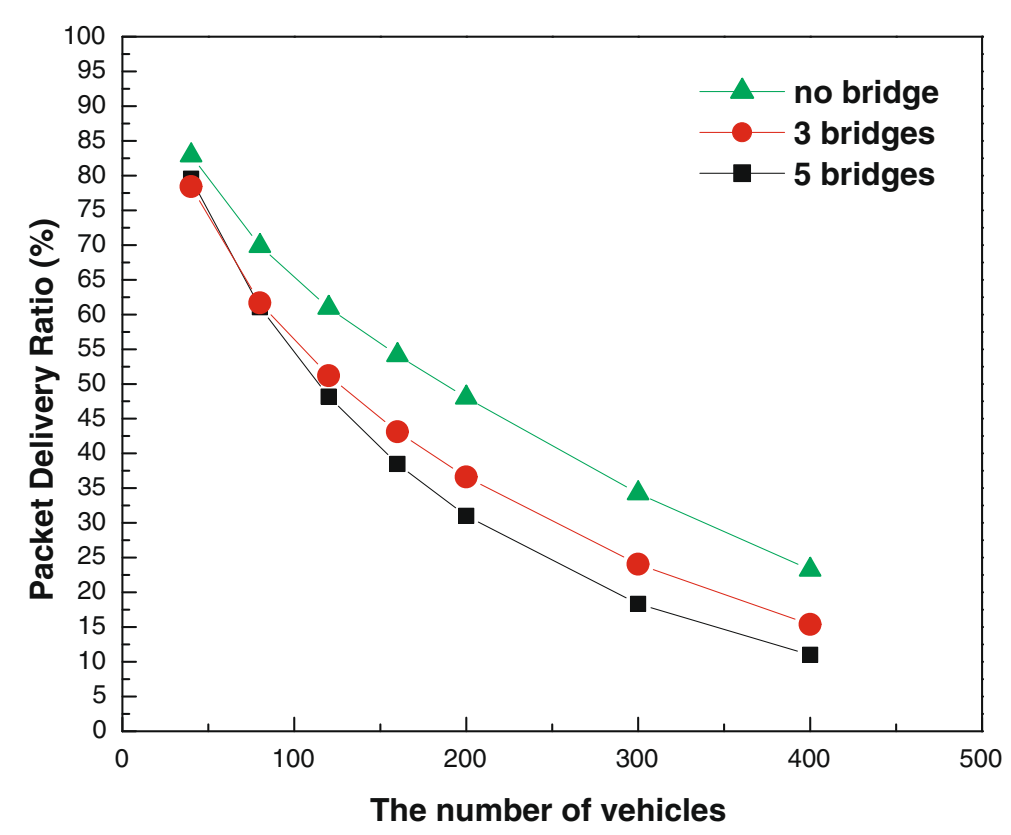

Fig. 10 PDR of STDMA according to the number of vehicles when time synchronization errors sporadically happen 
that a probability of this event increases with a vehicle density. Second, even with a centralized coordination, a fraction of vehicles inevitably choose the same slot if the number of vehicles in the range of each other exceeds the number of slots within NI. For example, in Fig. 3, the number of slots within NI is 90 , while the number of vehicles within a close vicinity (a circle with radius $500 \mathrm{~m}$ ) is 133 when there are 400 vehicles in a $2 \mathrm{mi}$ road. This implies that more than $32 \%$ of the vehicles in a close vicinity must choose the same time slot even with a centralized controller. Third, current STDMA lacks a mechanism for adapting configurable parameters to network conditions. As shown in Sections 4.4 and 4.5, careful selection of a physical transmission rate and that of CS threshold can improve PDR of STDMA by up to 40 and $150 \%$, respectively.

The main reason for long delay is that we just follow suggestions of the current form of STDMA, which are not tailored to vehicular safety applications. Specifically, the current STDMA suggests to configure NI to be comparable to beacon interval, of which typical value is $100 \mathrm{~ms}$ and becomes longer if integrated with congestion control. It is obvious that average delay of deterministic protocol like STDMA is almost half a period for slot assignment, which corresponds to NI in STDMA. Hence, a delay of STDMA is long in such NI setting.

\subsection{Guidelines for improvement}

In this subsection, we briefly introduce how to improve STDMA based on our observations and analysis. One solution that can be easily derived from our observations is to adapt system parameter configurations to network conditions (e.g., vehicle density, channel errors). Specifically, to reduce delay, we necessarily diminish NI. For example, if network designers consider services of which delay bound is $20 \mathrm{~ms}$ (e.g., pre-crash sensing), we suggest to configure NI comparable to $20 \mathrm{~ms}$ because the delay of STDMA is upper-bounded by NI. In addition, to improve PDR, we necessarily configure a physical transmission rate and CS threshold. More specifically, we set a low transmission rate in low vehicle density and a high transmission rate in high density; we should find optimal CS threshold in terms of PDR.

We should note that the adaptive rate selection might induce another problem. Specifically, the improvement of an adaptive rate selection comes from a customization of slot size to message transmission time. However, unorganized customization of slot size may induce heterogeneity of transmission rates among vehicles in the vicinity. The heterogeneity, in turn, causes slot misalignment, which can be a reason of packet collision. Fortunately, a cluster-based mechanism has benefits in making cluster members share the same parameters (e.g., transmission rate). Therefore, as a future work, we will propose a new mechanism that improves STDMA based on a clustering mechanism.

The second suggestion is to employ an organized slot schedule. This solution is derived from our analysis above: random nature in slot selection degrades performances of STDMA. It is straightforward that the organized schedule can diminish collisions, thereby improving PDR. However, the organized schedule must be accompanied by a central controller (e.g., RSU), of which implementation and management are costly [23]. To circumvent this problem, we suggest to exploit a clustering mechanism, by which an organized schedule is possible within a cluster (i.e., the schedules of vehicles in the vicinity can be organized).

To summarize, our suggestion for improvement are as follows.

- Adaptive selection of a physical transmission rate and a CS threshold (PDR)

- Adaptive selection of NI (delay)

- Organized schedule via clustering mechanism (PDR)

\subsection{Towards improving STDMA: dynamic configuration}

In this subsection, we propose a dynamic configuration to improve STDMA performance. Among three parameters mentioned in Section 5.2, we focus on CS threshold and NI because we can configure these two parameters in a distributed manner. Recall that dynamic configuration of a physical transmission rate could degrade performance if vehicles in the vicinity exploit different rates; thus, a centralized method is necessary to control the transmission rate over the network, such as clustering mechanism. However, clustering sometimes induce additional overhead; thus, configuration of a physical transmission rate must be accompanied by careful design of clustering mechanism. Therefore, we do not consider configuring a transmission rate in this paper ${ }^{9}$.

The goal of the dynamic configuration is to maximize PDR while guaranteeing the upper bound of delay. For this purpose, we configure NI and CS threshold as follows. First, we configure NI to be comparable to the smallest among delay bounds of supported services. This is because a delay is upper-bounded by NI. As the delay bounds do not vary with time, NI is configured at the start of the network and the configured value rarely changes with time. Second, we dynamically configure CS threshold because the the CS threshold maximizing PDR depends on channel conditions (e.g., channel noise and vehicle density), which frequently changes with time. For this purpose, vehicles must monitor the channel conditions periodically and find an optimal value of CS threshold for each channel conditions. Specifically, each vehicle measures the channel conditions and share the conditions with neighbors by piggybacking the conditions onto the periodic safety message [22]. To find optimal CS threshold, 


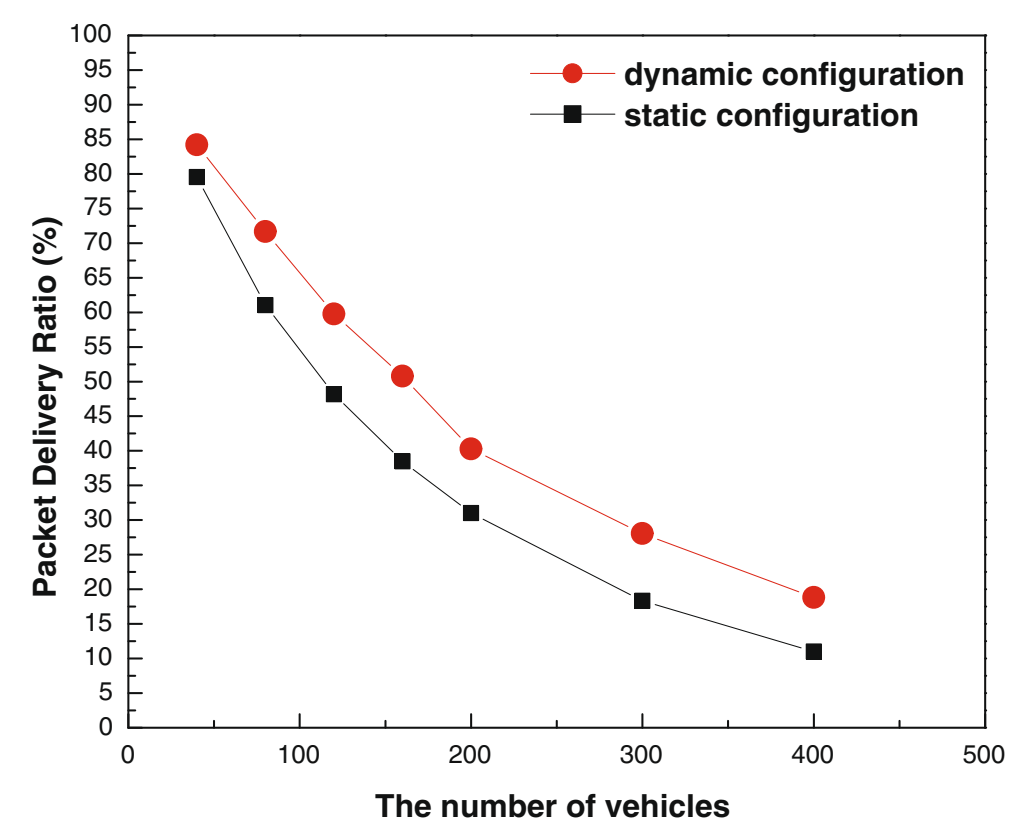

Fig. 11 Comparison of dynamic configurations with static configuration in terms of PDR

we exploit a table-driven approach. More specifically, each vehicle computes and preloads the optimal configuration table before driving ${ }^{10}$, in which optimal CS threshold is defined according to vehicle densities and channel noise; each vehicle finds optimal value in the table during driving.
To show the efficiency of dynamic configurations, we compare STDMA performances with dynamic configurations to those with typical static configurations. As depicted in Figs. 11 and 12, dynamic configurations improve PDR by up to $71 \%$ and reduce delay by up to $300 \%$ over static configurations. We

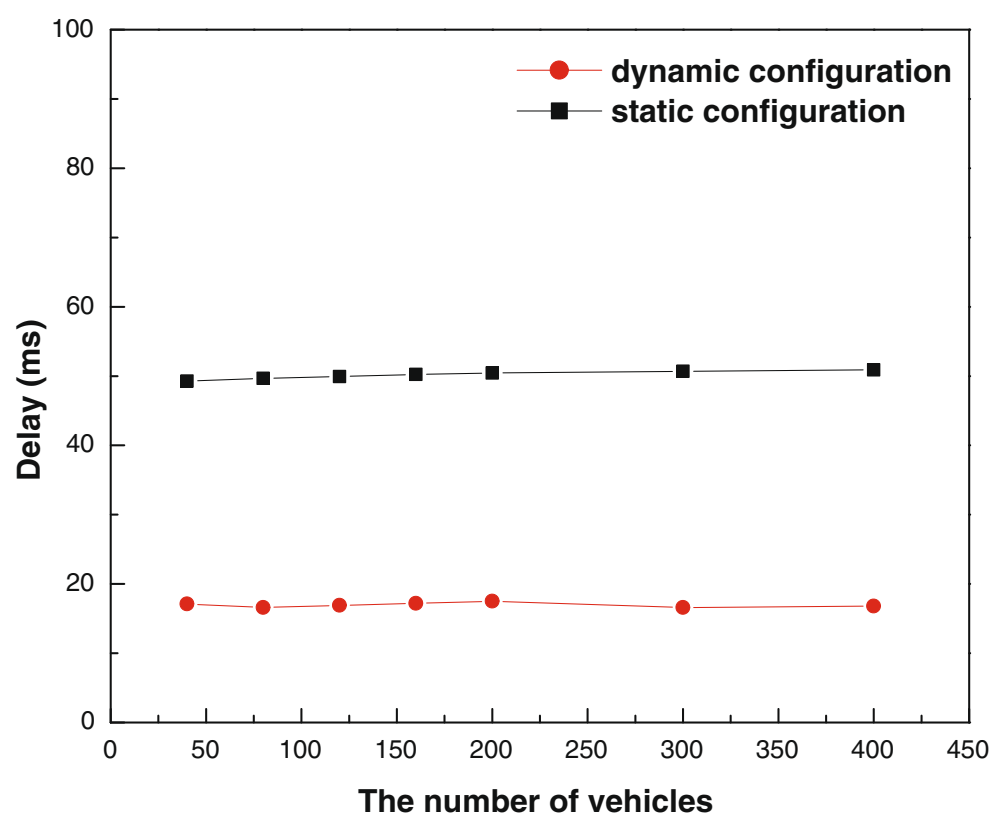

Fig. 12 Comparison of dynamic configurations with static configuration in terms of delay 
emphasize that this improvement comes from configuring only two parameters. Thus, we can further enhance STDMA performances by additionally configuring a physical transmission rate and integrating with an organized schedule, which will be our future work.

\section{Conclusions}

In this paper, we studied the performance of STDMA when it was adopted for vehicular safety applications. We found that STDMA guarantees an upper bound of delay, but PDR was not high enough to support vehicular safety applications, especially in high vehicle densities. Even more, we noticed that time synchronization error degraded PDR and the error was uncorrelated with delay. Through an in-depth analysis of simulation results, we provided rich set of hints for configuring system parameters-(1) we should decrease NI for reducing delay, (2) we should adapt a physical transmission rate to vehicle density for improving PDR, and (3) we should find optimal CS threshold for enhancing PDR. We also provided other suggestions for improvement. We hope that these results help to understand the characteristics of STDMA and encourage researchers to improve STDMA performance.

\section{Endnotes}

${ }^{1}$ Society for Automotive Engineers (SAE) [16] defines $100 \mathrm{~ms}$ to be a default generation period for periodic vehicle message. Moreover, the size of safety message is variable. For example, SAE 2735 specifies that the size of basic safety message (BSM) is variable [16] due to variable size of BSM payload part II ( $0 \sim 500$ bytes) [27]. Thus, we follow the size of safety message suggested in [4], which is generally accepted by industries. However, these values are just default settings.

${ }^{2} \mathrm{We}$ assume that only beacon with the same size is transmitted. However, message sizes can be various if several services are supported. In this case, we set the unit slot size that are comparable to the longest transmission duration.

${ }^{3}$ Default setting of SI is one fifth of NI [9-11]. However, this is just a default setting, which can change for further improvement.

${ }^{4} \mathrm{We}$ assume that thermal noise dominates the noise, which can be derived from Boltzman constant, bandwidth, and the temperature of typical wireless transceiver (http://en.wikipedia.org/wiki/Johnson\%E2 \%80\%93Nyquist_noise).
${ }^{5}$ The unit of SI is an integer. However, the integer can be translated into the continuous length of time by multiplying it with the unit slot of STDMA.

${ }^{6}$ Simple mathematical calculation reveals that the probability that the first event and the second event happen simultaneously is independent of SI, if we do not consider the case of selecting another slot due to busy slot selection at the first time.

${ }^{7}$ In STDMA, we can customize a unit time slot to the transmission rate. Thus, as the rate grows, the size of the unit slot, which in turn, increases the number of slots within NI.

${ }^{8}$ According to [17], the life-critical accidents happens at least once per year when the speed is above $35 \mathrm{mph}$. Moreover, the normal gap between two vehicles is around $1.5 \mathrm{~s}$, which is equivalent to $30 \mathrm{~m}$ gap [18]. Thus, as considering 4-lane road with $2 \mathrm{mi}$, we consider the number of vehicles can be up to 400 .

${ }^{9}$ Due to similar reason, we do not consider an organized schedule in this paper even if the organized schedule can further improve performances of STDMA.

${ }^{10}$ The naive approach to derive optimal parameters is brute-force search. Even if it takes much time to derive optimal parameters using brute-force approach, computing before driving needs not to be real-time [23].

\section{Acknowledgements \\ The authors wish to thank the Associate Editor and anonymous reviewers for their constructive suggestions and helpful comments.}

\section{Authors' contributions}

The author analyzed STDMA performances with practical errors that can occur in real worlds (e.g., time synchronization error) and evaluated the feasibility of STDMA to vehicular safety applications in such situations. The author confirmed that current form of STDMA is not feasible to vehicular safety applications. The author confirmed that proper setting of each configurable parameter really helps to improve STDMA performance and provided a set of hints for configurations based on observations. The author suggested guidelines for STDMA improvement to be suitable to vehicular safety applications.

\section{Competing interests}

The author declares that he has no competing interest.

Received: 26 April 2016 Accepted: 28 July 2016

Published online: 05 August 2016

\section{References}

1. U.S. Dept., Transportation National Highway Safety Admin. (Traffic Safety Facts, Washington, DC, USA, 2003)

2. IEEE Standard for Information Technology Telecommunications and Information Exchange Between Systems Local and Metropolitan Area Networks Specific Requirements; Part 11: Wireless LAN Medium Access Control (MAC) and Physical Layer (PHY) Specifications; Amendment 6: Wireless Access in Vehicular Environments. IEEE Std, 802.11p (2010)

3. Ahmed-Zaid, et al., Vehicle Safety Communications-Applications (VSC-A) Final Report. Technical Report (2011) 
4. S Sharafkandi, et al., in Proc. of IEEE Vehicular Network Conference (VNC). Using EDCA to Improve Vehicle Safety Message, (2012), pp. 70-77

5. J-H Lim, et al., in Proc. of IEEE Globecom. Interaction between EDCA and HCCA: Simulation Study of DSRC for Work Zone Safety, (2013), pp. 42-47

6. J-M Chung, et al., Time Coordinated V2I Communications and Handover for WAVE Networks. IEEE J. Sel. Area Commun. 29(3), 545-558 (2011)

7. H Aboubakr, et al., VeMAC: A TDMA-based MAC protocol for reliable broadcast in VANETs. IEEE Trans. Mob. Comput. 12(9), 1724-1736 (2013)

8. Recommendations ITU-R M.1371-1. Technical characteristics for universal shipborne automatic identification system using time division multiple access in the VHF maritime mobile band

9. A Alonso, et al., in Proc. of IEEE VTC. Throughput of Self-Organizing Time Division Multiple Access MAC Layer for Vehicular Networks Based on Measured SNR Time-Series, (2011)

10. K Bilstrup, in Proc. of ITS World Congress. On the Ability of the $802.11 \mathrm{p} \mathrm{MAC}$ Method and STDMA to Provide Predictable Channel Access, (2009)

11. VD Khainar, et al., Simulation based evaluation of highway road scenario between DSRC/802.11p MAC protocol and STDMA for vehicle-to-vehicle communication. J. Transp. Technol. 3, 88-104 (2013)

12. K Sjoberg, et al., in Proc.of IEEE VTC. How Severe is the Hidden Terminal Problem in VANETs when Using CSMA and STDMA? (2011)

13. K Sjoberg, et al., in Proc. of IEEE IWCMC. Delay and Interference Comparison of CSMA and Self-Organizing TDMA when Used in VANETs, (2011), pp. 1488-1493

14. T Gaugel, et al., in Proc.of IEEE VNC. In-Depth Analysis and Evaluation of Self-Organizing TDMA, (2013), pp. 79-86

15. The Network Simulator, ns-2. http://www.isi.edu/nsnam/ns

16. DRAFT SAE J2735 Dedicated Short Range Communications (DSRC) Message Set Dictionary. Rev 0.7, Jam (2006)

17. MC Taylor, et al., The effects of drivers' speed on the frequency of road accidents, Transport Research Lab (TRL) Report 421, Crowthorn, Berkshire, England, TRL Limited (2000)

18. D Sun, et al., in TRB 86th Annual Meeting Compendium of Papers CD-ROM. An In-Depth Analysis on Vehicle Following Gaps in Highway Work Zones: The Direct and Interdependent Impact of Leading Vehicle (Transportation Research Board of the National Academies, Washington, D.C., 2007)

19. T Sun, et al., Measurement Study of Path Capacity in 802.11 b Based Wireless Networks. (The Wireless Traffic Measurements and Modeling Workshop, Seattle, USA, 2005)

20. F Bai, et al., in Proc. of ACM MOBICOM. Toward Understanding Characteristics of Dedicated Short Range Communications (DSRC) From a Perspective of Vehicular Network Engineers, (2010), pp. 329-340

21. R Stanica, et al., in Proc. of IEEE MASS. Physical Carrier Sense in Vehicular Ad-Hoc Networks, (2011), pp. 580-589

22. J-H Lim, et al., Interplay between DSRC and TWWS: optimal strategy for safety message dissemination. IEEE J. Sel. Area Commun. (JSAC). 32(11), 2117-2133 (2014)

23. J-H Lim, et al., in Proc. of IEEE ICNC 2015. Safety Message Dissemination in NLOS Environments of Intersection Using TV White Space, (2015), pp. 451-455

24. Z Wanget, et al., in Proc.of ACM VANET. How Much of DSRC is Available for Non-Safety User? (2008), pp. 23-29

25. J Yin, et al., in Proc.of ACM VANET. Performance Evaluation of Safety Applications over DSRC Vehicular Ad Hoc Networks, (2004), pp. 1-9

26. JJ Olstam, et al., Comparison of Car-Following Models, VTI Meddelande 960A. (Swedish National Road and Transport Research Institute, Linkping, Sweden, 2004)

27. J Kenney, Dedicated Short-Range Communications (DSRC) Standards in the United States. Proc. IEEE. 99(7), 1162-1182 (2011)

28. T Meng, et al., Spatial reusability-aware routing in multi-hop wireless networks. IEEE Trans. Comput. 65(1), 244-255 (2016)

29. XM Zhang, et al., Interference-based topology control algorithm for delay-constrained mobile Ad hoc networks. IEEE Trans. Mob. Comput. 14(4), 742-754 (2015)

30. P Li, et al., Reliable multicast with pipelined network coding using opportunistic feeding and routing. IEEE Trans. Parallel Distrib. Syst. 25(12), 3264-3273 (2014)

31. Q Jing, et al., Security of the Internet of Things: perspectives and challenges. Springer Wirel. Netw. 20(8), 2481-2501 (2014)
32. D López-Pérez, et al., On distributed and coordinated resource allocation for interference mitigation in self-organizing LTE networks. IEEE/ACM Trans. Networking. 21(4), 1145-1158 (2013)

33. $Y$ Zeng, et al., Directional routing and scheduling for green vehicular delay tolerant networks. Springer Wirel. Netw. 19(2), 161-173 (2013)

34. M Youssef, et al., Routing metrics of cognitive radio networks: a survey. IEEE Commun. Surv. Tutor. 16(1), 92-109 (2014)

35. M-K Jiau, et al., Multimedia services in cloud-based vehicular networks. IEEE Intell. Transp. Syst. Mag. 7(3), 62-79 (2015)

36. J Wan, et al., VCMIA: a novel architecture for integrating vehicular cyber-physical systems and mobile cloud computing. ACM/Springer Mob. Netw. Appl. 19(2), 153-160 (2014)

37. TWang, et al., Survey on channel reciprocity based key establishment techniques for wireless systems. Springer Wirel. Netw. 21(6), 1835-1846 (2015)

38. J Zhou, et al., Secure and privacy preserving protocol for cloud-based vehicular DTNs. IEEE Trans. Inf. Forensics Secur. 10(6), 1299-1314 (2015)

39. J Liu, et al., A survey on position-based routing for vehicular ad hoc networks. Springer Telecommun. Syst. 62(1), 15-30 (2016)

40. W Viriyasitavat, et al., Vehicular communications: survey and challenges of channel and propagation models. IEEE Veh. Technol. Mag. 10(2), 55-66 (2015)

41. H Yang, et al., Provably secure three-party authenticated key agreement protocol using smart cards. Elsevier Comput. Netw. 58, 29-38 (2014)

42. J Wan, et al., loT Sensing framework with inter-cloud computing capability in vehicular networking. Electron. Commer. Res. 14(3), 389-416 (2014)

43. B Liu, et al., Toward incentivizing anti-spoofing deployment. IEEE Trans. Inf. Forensics Secur. 9(3), 436-450 (2014)

44. J Liu, et al., Leveraging software-defined networking for security policy enforcement. Elsevier Inf. Sci. 327, 288-299 (2016)

45. J Wan, et al., Context-aware vehicular cyber-physical systems with cloud support: architecture, challenges and solutions. IEEE Commun. Mag. 52(8), 106-113 (2014)

46. Z Yan, et al., A security and trust framework for virtualized networks and software defined networking. Wiley Secur. Commun. Netw. (2015). onlinelibrary.wiley.com/doi/10.1002/sec.1243/full

\section{Submit your manuscript to a SpringerOpen ${ }^{\circ}$ journal and benefit from:}

- Convenient online submission

- Rigorous peer review

- Immediate publication on acceptance

- Open access: articles freely available online

- High visibility within the field

- Retaining the copyright to your article

Submit your next manuscript at $>$ springeropen.com 